\title{
A Comparison of Filtering Techniques for Image Quality Improvement in Computed Tomography
}

\author{
Dr. Sachin D. Ruikar, Ms. Vrushali N. Raut \\ SinhgadAcademy of Engineering, E \& TC Deparment, University of Pune, India \\ ruikaretcdept@gmail.com \\ Sinhgad Academy of Engineering, E \& TC Deparment, University of Pune, India \\ vrushali_ss@yahoo.com
}

\begin{abstract}
Computed Tomography (CT) is an important and most common modality in medical imaging. In CT examinations there is trade off between radiation dose and image quality. If radiation dose is decreased, the noise will unavoidably increase degrading the diagnostic value of the CT image and ifthe radiation dose is increased, the associated risk of cancer also increases especially in paediatric applications. Image filtering techniques perform image pre-processing to improve the quality of images. These techniques serve two major purposes. One is to maintain low radiation dose and another is to make subsequent phases of image analysis like segmentation or recognition easier or more effective. This paper presents the effect of noise reduction filter on CT images particularly that of anisotropic diffusion filter and Gaussian filter in combination with Prewitt operator. Anisotropic diffusion is Selective and nonlinear filtering technique which filters an image within the object boundaries and not across the edge orientation. Simulation results have shown that the anisotropic diffusion filter can effectively smooth noisy background, yet well preserve edge and fine details in the restored image. Gaussian filter smoothens the image while Prewitt operator detects the edges, so the combination of Gaussian filters and Prewitt operator works like a nonlinear filter.Thus these two filtering techniques improve an image quality and allow use of low dose CT protocol.
\end{abstract}

\section{Keywords}

Anisotropic diffusion, computed tomography, image quality, non linear filter, radiation dose

\section{Academic Discipline And Sub-Disciplines}

Education: Electronics \& Telecommunication

\section{SUBJECT CLASSIFICATION}

Image Processing

\section{TYPE (METHOD/APPROACH)}

Experimental using MATLAB, Literary Analysis.

\section{Council for Innovative Research}

\author{
Peer Review Research Publishing System
}

\section{Journal: INTERNATIONAL JOURNAL OF COMPUTERS \& TECHNOLOGY}

\author{
Vol 7, No 3




\section{INTRODUCTION}

During the last few years there has been a dramatic increase in the number of CT examinations performed worldwide. The introduction of multislice CT systems offering helical 3D scan techniques has allowed a wider range of diagnostic examinations to be performed. Computed tomography (CT) is a radiographic inspection method that generates a 3-D image of the inside of an object from a large series of 2-D images taken on a cross sectional plane of the same object [1]. CT overcomes the problem of overlying tissues by scanning thin slices of the body with a narrow X-ray beam (produced by an X-ray tube), which rotates around the body of the stationary patient. The X-rays passing through the body of the patient is picked up by a row of detectors. The tube and the detectors are positioned on opposite sides of a ring (gantry) rotating around the patient. The CT image is derived from a large number of systematic observations at different viewing angles; then, a 3-D image is reconstructed from the data by means of a processor [2]-[4].

While image quality has always been a concern for the physics community, clinically acceptable image quality has become even more of an issue as strategies to reduce radiation dose - especially to pediatric patients- become a larger focus. Dose reduction in pediatric patients is critical, due to the relatively high absorption in the small body volume; the greater sensitivity of rapidly growing tissue to radiobiological damage [5]. One study suggested that as much as $1.5-2 \%$ of cancers may eventually be caused by the radiation dose currently used in CT examinations [6].

The visualization of the anatomic structures by means of CT is affected by two effects, namely, blurring, which reduces the visibility of small object, and noise, which reduces the visibility of low-contrast objects. Sources of blurring in CT include the size of the sampling aperture, the size of the voxels, and the reconstruction filter selected. Noise is caused by the variation in attenuation coefficients between voxels. Use of small voxels and edge-enhancing filters helps reduce blurring and improve visibility of fine details. However, small voxels absorb fewer photons and therefore result in increased noise.Many technical factors contribute to the intensity dose in CT like tube current and gantry rotation time, tube voltage peak, Pitch. The most commonly manipulated parameter to manage the radiation dose is the tube current [7]. Generally, in CT examinations, a high radiation dose results in high-quality images. A lower dose leads to the increase in image noise and results in unsharp images. Although reduction in radiation dose is an important exercise, maintaining high quality of a diagnostic imaging study is also essential to provide an accurate and definitive diagnosis.

CT images are intrinsically noisy, and this poses significant challenges for image interpretation. CT noise affects the visibility of low-contrast objects. In the CT image, the primary contributor to the total noise is the quantum noise and the other noise can be electronic noise [8]-[9]. By using filters it is possible to obtain, at the same time, high-quality images with low-dose radiation. As evident in the literature, noise modeling and the way to reduce it are common problems in most imaging applications. In many image processing applications, a suitable denoising phase is often required before any relevant information could be extracted from analyzed images [10]-[12].

\section{MATERIALS AND METHODS}

The CT images (512 × 512 pixels) acquired are in Digital Imaging and Communications in Medicine (DICOM) format, which represents the standard in radiology and cardiology imaging industry for data. The examined images are acquired by means of a helical CT scanner.

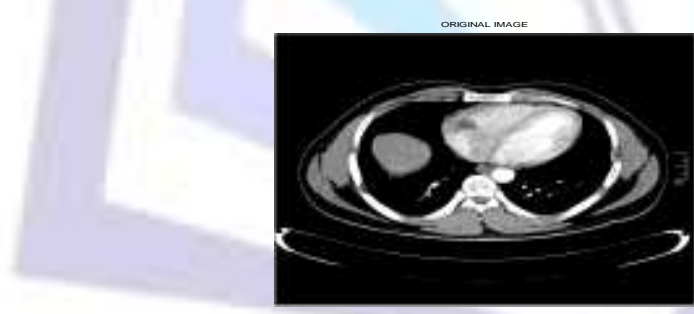

(a)

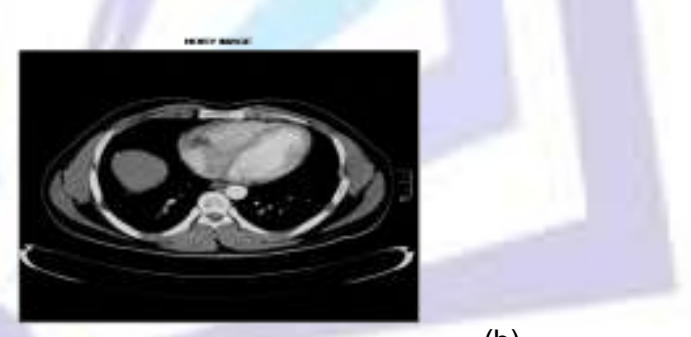

(b)

Figure.1. (a) Original CT image obtained with a high dose of radiation. (b) Emulated low-dose CT image obtained by corrupting the high-dose CT image.

Each image is corrupted by additive zero-mean white Gaussian noise to simulate a low-dose CT image. For this, the tube current level must be reduced to get noisy image. Figure.1. shows original high-dose chest image [Figure. 1(a)] and its noisy version [Figure. 1(b)].

\section{Anisotropic Diffusion}

Anisotropic diffusion, also called Perona-Malik diffusion, is a technique aiming at reducing image noise without removing significant parts of the image content, typically edges, lines or other details that are important for the interpretation of the image. This diffusion process is a linear and space-invariant transformation of the original image. Anisotropic diffusion is a generalization of this diffusion process.Anisotropic diffusion can be used to remove noise from digital images without blurring edges. If the diffusion coefficient is chosen as an edge seeking function, then the quality of image improves as the edges are preserved while noise is removed from the image [13].

Anisotropic diffusion is a selective and nonlinear filtering technique that improves an image quality, removing the noise while preserving and even enhancing details. The anisotropic diffusion process employs the diffusion coefficients to 
determine the amount of smoothing that should be applied to each pixel of the image. The diffusion process is based on an iterative method, and it is described by means of the following diffusion equation.

$$
I_{i, j}^{t+1}=I_{i, j}^{t}+\lambda\left(C_{N} \nabla_{N} I+C_{S} \nabla_{S} I+C_{E} \nabla_{E} I+C_{W} \nabla_{W} I+C_{N E} \nabla_{N E} I+C_{N W} \nabla_{N W} I+C_{S E} \nabla_{S E} I+C_{S W} \nabla_{S W} I\right)_{i, j}^{t}
$$

Where $I_{i, j}^{t+1}$ is the intensity of the pixel at position $\mathrm{i}, \mathrm{j}$ and at the $\mathrm{t}^{\text {th }}$ iteration; $\mathrm{C}_{\mathrm{N}}, \mathrm{C}_{\mathrm{S}}, \mathrm{C}_{\mathrm{E}}, \mathrm{C}_{\mathrm{W}}, \mathrm{C}_{\mathrm{NE}}, \mathrm{C}_{\mathrm{NW}}, \mathrm{C}_{\mathrm{SE}}, \mathrm{C}_{\mathrm{SW}}$ are the diffusion coefficients in the eight directions (north, south, east, west, north-east, north-west, south-east, south-west) parameters $\nabla_{\mathrm{N}} \mathrm{I}, \nabla_{\mathrm{S}}, \nabla_{\mathrm{E}} \mathrm{I}, \nabla_{\mathrm{W}}, \nabla_{\mathrm{NE}} \mathrm{I}, \nabla_{\mathrm{NW}}, \nabla_{\mathrm{SE}} \mathrm{I}, \nabla_{\mathrm{SW}} \mathrm{l}$ are the nearest-neighbour differences of intensity in the eight directions; and $\lambda$ represents a coefficient that assures the stability of the model, ranging in the interval [0-1/7]. The initial condition $(\mathrm{t}=0)$ of the diffusion equation is the intensity pixels of the original image. It applies the law of diffusion on pixel intensities to smooth textures in an image. A threshold function is used to prevent diffusion to happen across edges, and therefore it preserves edges in the image. The diffusion coefficients are updated at every iteration as a function of intensity gradient. One of the following functions, either $\mathrm{C}_{\mathrm{D} 1}$ or $\mathrm{C}_{\mathrm{D} 2}$ can be used for coefficient calculation:

$$
C_{D 1}=e^{\left(-\frac{\left|\nabla_{D} I\right|}{K}\right)^{2}} \quad C_{D 2}=\frac{1}{1+\left(\frac{\nabla_{D} I}{K}\right)^{2}} \quad D=[N, S, E, W, N E, N W, S E, S W](2)
$$

Where $\mathrm{K}$ is a control parameter. The first function favours high-contrast edges over low contrast edges, while the second emphasizes wider regions over smaller regions. A proper choice of the diffusion function not only preserves but also enhances the edges. This function monotonically decreases with the increase in gradient intensity $\nabla$. The control parameter should produce maximum smoothing where noise is supposed to be present. The value of $\mathrm{K}$ can be calculated to find the maximum value of diffusion flow and take it to be equal to the noise level. The following $\mathrm{K}$ values are obtained for two diffusion functions (2):

$$
K_{1}=\sqrt{2} \cdot \sigma_{n} K_{2}=\sigma_{n}
$$

Where $\sigma_{n}$ is the standard deviation of the noise calculated in the noisy image background. The estimation of the noise level in a corrupted image is based on the calculation of the standard deviation of the pixels in the background. So, the pixel indexes of the original image background, corresponding to the zones where there is no signal $\left(\mathrm{l}_{\mathrm{i}, \mathrm{j}}=0\right)$, have been calculated. Then, these indexes are used to calculate the standard deviation in the noisy image. Actually noise is nonstationary so $\mathrm{K}$ must be calculated as a function of local noise characteristics. The noise is assumed to be statistically independent of the original image. We considered the differences in intensity in the eight directions, i.e.

$$
\begin{aligned}
& D_{N_{i, j}}=\frac{1}{\sqrt{2}}\left(I_{i+1, j}-I_{i, j}\right)=\frac{1}{\sqrt{2}}\left(\nabla_{N} I\right)_{i, j} \\
& D_{S_{i, j}}=\frac{1}{\sqrt{2}}\left(I_{i-1, j}-I_{i, j}\right)=\frac{1}{\sqrt{2}}\left(\nabla_{S} I\right)_{i, j} \\
& D_{E_{i, j}}=\frac{1}{\sqrt{2}}\left(I_{i, j+1}-I_{i, j}\right)=\frac{1}{\sqrt{2}}\left(\nabla_{E} I\right)_{i, j} \\
& D_{W_{i, j}}=\frac{1}{\sqrt{2}}\left(I_{i, j-1}-I_{i, j}\right)=\frac{1}{\sqrt{2}}\left(\nabla_{W} I\right)_{i, j} \\
& D_{N E_{i, j}}=\frac{1}{\sqrt{2}}\left(I_{i+1, j+1}-I_{i, j}\right)=\frac{1}{\sqrt{2}}\left(\nabla_{N E} I\right)_{i, j} \\
& D_{S E_{i, j}}=\frac{1}{\sqrt{2}}\left(I_{i-1, j+1}-I_{i, j}\right)=\frac{1}{\sqrt{2}}\left(\nabla_{S E} I\right)_{i, j} \\
& D_{N W_{i, j}}=\frac{1}{\sqrt{2}}\left(I_{i+1, j-1}-I_{i, j}\right)=\frac{1}{\sqrt{2}}\left(\nabla_{N W} I\right)_{i, j} \\
& D_{S W_{i, j}}=\frac{1}{\sqrt{2}}\left(I_{i-1, j-1}-I_{i, j}\right)=\frac{1}{\sqrt{2}}\left(\nabla_{S W} I\right)_{i, j}
\end{aligned}
$$

The noise variance of the sum of two independent noisy signals is the sum of the noise variances of the two components. Therefore, it can easily be shown that the variance of the noise is not affected by the operations in (4), because the noise is assumed to be a white signal, i.e., different pixels are not correlated. Then, the noise variances of $I, D_{N}, D_{S}, D_{E}, D_{W}$, $D_{N E}, D_{S W}, D_{S E}, D_{N W}$ are the same. To estimate the local noise standard deviation, a subimage of size $\mathrm{M}(\mathrm{M}=2 \mathrm{~m}+1)$ is considered, with following relationship.

$$
\sigma_{n_{D_{i, j}}}=\sqrt{\frac{1}{M^{2}-1} \sum_{r=-m}^{m} \sum_{k=-m}^{m}\left(D_{D i+m, i+k}-\mu_{D_{i, j}}\right)^{2}}
$$




$$
\mu_{D_{i, j}}=\frac{1}{M^{2}} \sum_{r=-m}^{m} \sum_{k=-m}^{m} D_{D i+m, i+k} \quad D=[N, S, E, W, N E, S E, N W, S W]
$$

Local mean $\mu_{D_{i, j}}$ is taken into account. Even if the global noise mean is zero, locally, the mean is usually nonzero [14]. The estimated local standard deviation is replaced in (3), obtaining eight $\mathrm{K}$ values for each diffusion function.

\section{Gaussian Filter with Prewitt Operator}

To reduce the noise effect, different low-pass filters have largely been used in medical image analysis, but they have the disadvantage to introduce blurring edges. In fact, all smoothing filters, while smoothing out the noise, also removes high frequency edge features by degrading the localization and the contrast. Therefore, it is necessary to balance the trade-off among noise suppression, image deblurring, and edge detection. To this aim, a low-pass filter combined with an edge detector operator is proposed. In particular, Gaussian filter which smoothen the noise is combined with Prewitt operator which is used for edge identification.

2.1Gaussian Filter: A Gaussian filter smoothens an image by calculating weighted averages in a filter box. In electronics and signal processing, a Gaussian filter is a filter whose impulse response is a Gaussian function. Gaussian filters are designed to give an overshoot to a step function input while minimizing the rise and fall time. Gaussian filter modifies the input signal by convolution with a Gaussian function; this transformation is also known as the Weierstrass transform [15].Gaussian smoothing is also used as a pre-processing stage in computer vision algorithms in order to enhance image structures at different scales. Gaussian filtering is the filtering with am $x n$ mask. The weights are computed according to a Gaussian function.

$$
g(x, y)=\frac{1}{2 \pi \sigma^{2}} \cdot e^{\frac{-\left(x^{2}+y^{2}\right)}{2 \sigma^{2}}}
$$

Where $\sigma$ is user defined.

2.2Prewitt Operator:The Prewitt operator is used in image processing, particularly within edge detection algorithms. Technically, it is a discrete differentiation operator, computing an approximation of the gradient of the image intensity function. At each point in the image, the result of the Prewitt operator is either the corresponding gradient vector or the norm of this vector. The Prewitt operator is based on convolving the image with a small, separable, and integer valued filter in horizontal and vertical direction and is therefore relatively inexpensive in terms of computations.

In simple terms, the operator calculates the gradient of the image intensity at each point, giving the direction of the largest possible increase from light to dark and the rate of change in that direction. The result therefore shows how "abruptly" or "smoothly" the image changes at that point and therefore how likely it is that that part of the image represents an edge, as well as how that edge is likely to be oriented. In practice, the magnitude (likelihood of an edge) calculation is more reliable and easier to interpret than the direction calculation.

Mathematically, the gradient of a two-variable functionis at each image point a $2 \mathrm{D}$ vector with the components given by the derivatives in the horizontal and vertical directions. At each image point, the gradient vector points in the direction of largest possible intensity increase, and the length of the gradient vector corresponds to the rate of change in that direction. This implies that the result of the Prewitt operator at an image point which is in a region of constant image intensity is a zero vector and at a point on an edge is a vector which points across the edge, from darker to brighter values. The operator uses two $3 \times 3$ kernels which are convolved with the original image to calculate approximations of the derivatives one for horizontal changes, and one for vertical. If $A$ is a source image, and $G_{x}$ and $G_{y}$ are two images which at each point contain the horizontal and vertical derivative approximations, the latter are computed as:

$$
G_{X}=\left[\begin{array}{lll}
-1 & 0 & +1 \\
-1 & 0 & +1 \\
-1 & 0 & +1
\end{array}\right] * A \& G_{Y}=\left[\begin{array}{ccc}
+1 & +1 & +1 \\
0 & 0 & 0 \\
-1 & -1 & -1
\end{array}\right] * A
$$

Where ${ }^{*}$ here denotes the 2-dimensional convolution operation. Since the Prewitt kernels can be decomposed as the products of an averaging and a differentiation kernel, they compute the gradient with smoothing. The $x$-coordinate is defined here as increasing in the "right"-direction, and the $y$-coordinate is defined as increasing in the "down"-direction. At each point in the image, the resulting gradient approximations can be combined to give the gradient magnitude \& edge direction, using [16]:

Edge Magnitude $=\sqrt{G_{x}^{2}+G_{y}^{2}}$
Edge Direction $=\tan ^{-1}\left[\frac{G_{y}}{G_{x}}\right]$

\section{RESULTS}

The effect of noise addition on the original images is evaluated by calculating the relative RMS error by using following formula, 


$$
e_{R M S}=\sqrt{\frac{\sum_{i=1}^{r} \sum_{j=1}^{c}\left(I_{i, j}-I_{o i, j}\right)^{2}}{\sum_{i=1}^{r} \sum_{j=1}^{c}\left(I_{o i, j}\right)^{2}}}
$$

Where $I_{0}$ is the original high-dose image, $I$ is the original image corrupted by Gaussian noise, and $R$ and $C$ are the row and column numbers, respectively. The reduction in noise is calculated for both the filtering techniques. Several simulations have been used to set up the filter parameters. A first set of tests has been carried out to compare the performances of the filter obtained by calculating the diffusion coefficients by using any of the two functions (2). The test results show that the first function produces slightly better performance than the second function. Then simulations have been performed to identify the number of iterations for the diffusion process.

Using an anisotropic filter, it is possible to decrease the RMS error to about $7 \%$ which was $13 \%$ in the noisy image. The filter obtained by combining Gaussian and Prewitt were tested. This technique allows decreasing the average relative error to $10 \%$. Finally, the performances of the Gaussian-Prewitt and anisotropic filters have been compared. Figure.2. shows an example of the performance of both anisotropic filtering and combination of Gaussian filter and Prewitt operators. Table I shows the results of the simulation tests on fifteen CT images.
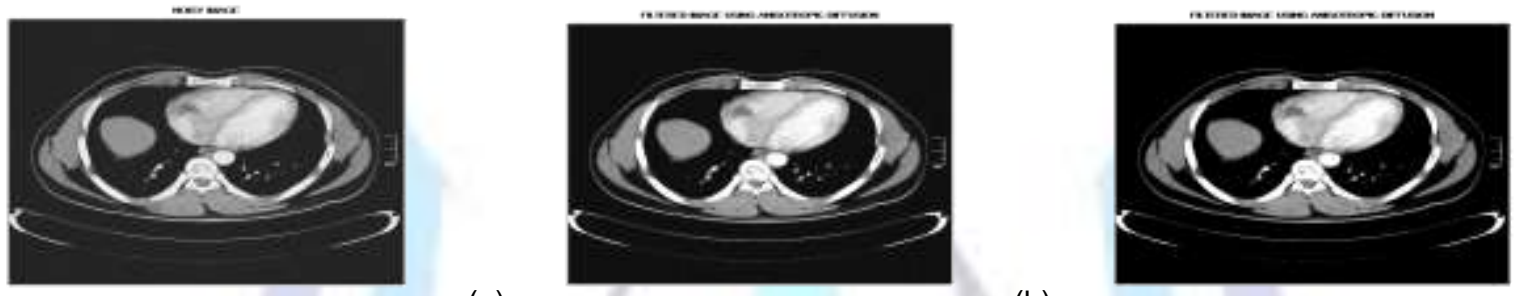

(a)

(b)

(c)

Figure. 2. (a) Noisy image. (b) Filtered noisy image obtained by combining Gauss and Prewitt operator (c) Filtered noisy image obtained using an anisotropic filter with three iterations

TABLE I

Results of the tests carried out on 15 CT images. In the three columns, the relative rms error for noisy images,for filtered images using gauss and prewitt filter, and for filtered images using anisotropic filter are listed

\begin{tabular}{|c|c|c|c|}
\hline Images & Noisy Images & Gauss-Prewitt filter & Anisotropic filter \\
\hline Image No.1 & $13.3 \%$ & $10.1 \%$ & $5.1 \%$ \\
\hline Image No.2 & $12.9 \%$ & $9.0 \%$ & $6.8 \%$ \\
\hline Image No.3 & $13.1 \%$ & $9.8 \%$ & $7.1 \%$ \\
\hline Image No.4 & $13.6 \%$ & $10.6 \%$ & $6.7 \%$ \\
\hline Image No.5 & $12.7 \%$ & $9.5 \%$ & $7.1 \%$ \\
\hline Image No.6 & $13.3 \%$ & $10.0 \%$ & $7.4 \%$ \\
\hline Image No.7 & $13.3 \%$ & $9.9 \%$ & $6.5 \%$ \\
\hline Image No.8 & $13.0 \%$ & $10.2 \%$ & $7.1 \%$ \\
\hline Image No.9 & $12.9 \%$ & $10.1 \%$ & $7.3 \%$ \\
\hline Image No.10 & $13.5 \%$ & $10.5 \%$ & $6.2 \%$ \\
\hline Image No.11 & $13.1 \%$ & $10.5 \%$ & $6.6 \%$ \\
\hline Image No.12 & $12.8 \%$ & $9.9 \%$ & $7.8 \%$ \\
\hline Image No.13 & $12.7 \%$ & $10.1 \%$ & $7.4 \%$ \\
\hline Image No.14 & $13.2 \%$ & $10.8 \%$ & $\mathbf{7 . 0 \%}$ \\
\hline Image No.15 & $13.3 \%$ & $10.3 \%$ & $\mathbf{1 0 . 0} \%$ \\
\hline Average & $\mathbf{1 3 . 0 \%}$ & & \\
\hline
\end{tabular}

\section{CONCLUSION}

In this paper, an analysis of denoising techniques applied to CT images is presented to increase the reliability of CT examinations obtained with low-dose radiation.Several simulations have been carried out to choose the best filter 
parameters. Using anisotropic filter, it is possible to decrease RMS error to about $7 \%$ and by using Gauss-Prewitt operator the error can be reduced to $10 \%$ which was $13 \%$ in noisy images. This way, it is possible to decrease the relative error in CT images \& to improve image quality.

\section{ACKNOWLEDGMENT}

This work has been carried out with the help of images and technical material that is supplied by the Prism medical diagnostics, Solapur, India.

\section{REFERENCES}

[1] F. Attivissimo, G. Cavone "A technique to improve the image quality in computer tomography". Transactions on instrumentation and measurement, Vol. 59, No. 5, May 2010.

[2] C. J. Garvey and R. Hanlon, "Computed tomography in clinical practice," BMJ, vol. 324, no. 7345, pp. 1077-1080, May 2002.

[3] H. Palmans and F. Verhaegen, "Assigning non elastic nuclear interaction cross sections to Hounsfield units for Monte Carlo treatment planning of proton beams," Phys. Med. Biol., vol. 50, no. 5, pp. 991-1000, Mar. 2005.

[4] M. Prokop, M. Galanski, A. J. van der Molen, and C. Schaefer-Prokop, Spiral and Multislice Computed Tomography of the Body. Stuttgart, NY: Thieme, 2003.

[5] J. Wesselling, R. Esseling, R. Raupach, S. Fockenberg, N. Osada, J. Gerb, W. Heindel, and R. Fischbach, "The effect of dose reduction and feasibility of edge-preserving noise reduction on the detection of liver lesions using MSCT," Eur. Radio I., vol. 17, no. 7, pp. 1885-1891, Jul. 2007.

[6] A. B. Smith, W. P. Dillon, R. Gould, and M. Wintermark, "Radiation dose-reduction strategies for neuro radiology CT protocols," Amer. J. Neuro radio I., vol. 28, no. 9, pp. 1628-1632, Oct. 2007.

[7] M. F. McNitt-Gray, "AAPM/RSNA physics tutorial for residents: Topics in CT; radiation dose in CT," Radio Graphics, vol. 22, no. 6,pp. 1541-1553, Nov./Dec. 2002.

[8] M. Kachelrie and W. A. Kalender, "Pre sampling, algorithm, and noise: Considerations for CT in particular for medical imaging in general," Med. Phys., vol. 32, no. 5, pp. 1321-1334, May 2005.

[9] P. Sprawls, "CT image detail and noise," Radio Graphics, vol. 12, no. 5,pp. 1041-1046, Sep. 1992.

[10] P. Gravel, G. Beaudoin, and J. A. De Guise, "A method for modelling noise in medical images," IEEE Trans. Med. Imaging, vol. 23, no. 10,pp. 1221-1232, Oct. 2004.

[11] T. Lei and W. Sewchand, "Statistical approach to X-ray CT imaging and its applications in imaging analysis-Part I: Statistical analysis of X-ray CT imaging," IEEE Trans. Med. Imaging, vol. 11, no. 1, pp. 53-61, Mar. 1992.

[12] H. Lu, I.-T. Hsiao, X. Li, and Z. Liang, "Noise properties of low-dose CT projections and noise treatment by scale transformations," in Conf. Rec. IEEE Nucl. Sci. Symp., Nov. 2001, vol. 3, pp. 1662-1666.

[13] G. Andria, F. Attivissimo, G. Cavone, A. M. L. Lanzolla, T. Magli, and M. Spadavecchia, "Denoising filter to improve the quality of CT images," in Proc. I2MTC, Singapore, May 5-7, 2009, pp. 947-950.

[14] R. San-Jose, M. Martin-Fernandez, P. Caballero-Martinez, C. Alberola-Lopez, and J. Ruiz-Alzola, "A theoretical framework to three dimensional ultrasound reconstruction from irregularly-sample data," Ultrasound in Medicine and Biology, no. 2, pp. 255-269, 2003.

[15] K. Krissian, "A New Variational Image Restoration Applied to 3D Angiographies," in IEEE W. on Var. and Level Set Meth. in Comp. Vision July 2001, pp. 65-72.

[16] X. Zong, A. F. Laine, and E. A. Geiser, "Speckle reduction and contrast enhancement of echocardiograms via

[17] multiscale nonlinear processing," IEEE Trans. on Medical Imaging, vol. 17, no. 4, pp. 532-540, 1998. 


\section{AUTHOR’ BIOGRAPHY WITH PHOTO}
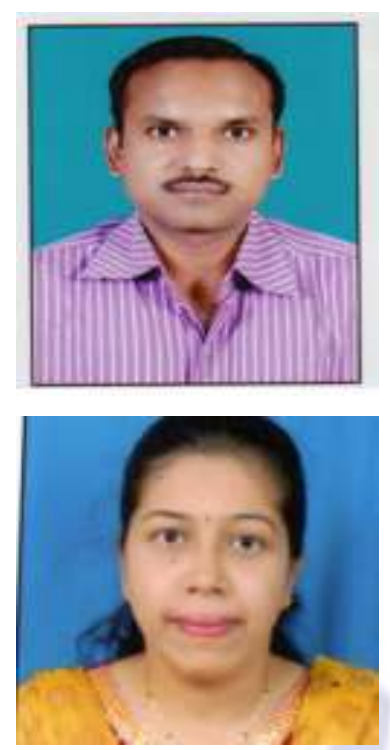

RuikarSachin D has received Graduate degree in Electronics and Telecommunication from Government Engineering College, Aurangabad, under the aegis of Dr. B. A. M. U., Aurangabad, in 1998. He has received postgraduate degree in Electronics and Telecommunication Engineering from Government Engineering College, Pune University, India in 2002. He has completed PhD in Electronics under Shri Guru Gobind Singh Institute of Engineering Technology, SRTMU Nanded, in 2013. Presently, he is working as Associate Professor in Electronics and Telecommunication at Sinhgad Academy of Engineering, Pune. His research interests include image denoising with wavelet transforms, image fusion, image painting and Image Superresolution.

VrushaliRauthas received Graduate degree in Electronics andTelecommunication from T.P.C.T's College of Engineering, Osmanabad under the aegis of Dr. B. A. M. U., Aurangabad, in 1999. Presently she is persuing masters of Engineering from Sinhgad Academy of Engineering, Pune. Her research interests include image denoising with different kind of filters ;linear\& nonlinear,Image quality improvement.

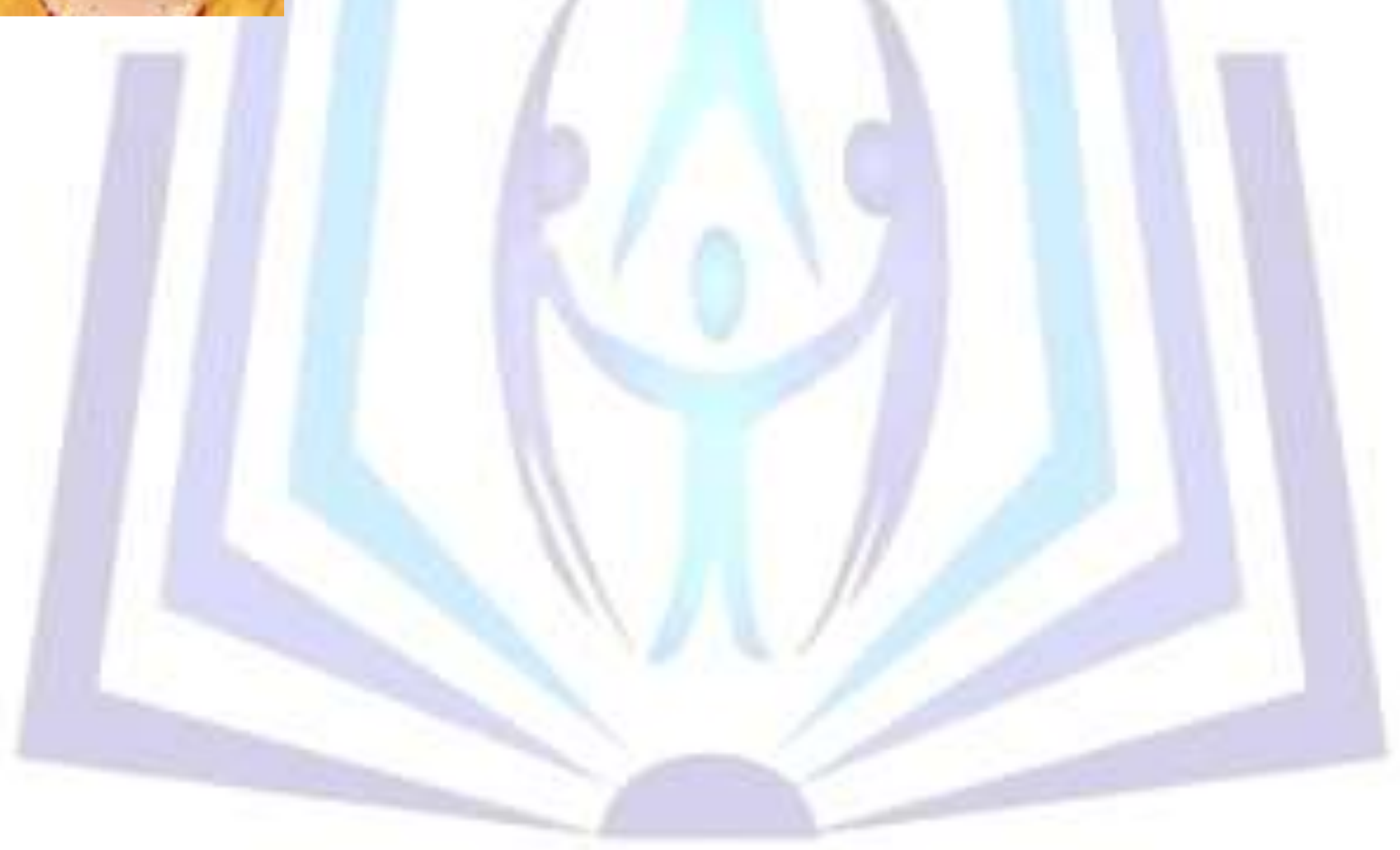

